\title{
Performance Evaluation of Zrp Star and Dsr under Vanet's
}

\author{
Harjit Singh, Manju Bala*, Manoj Kumar** \\ (Department of Computer Science and Engineering, CTIEMT, Shahpur, Jalandhar, Punjab,INDIA, \\ *(Department of Computer Science and Engineering, CTIEMT, Shahpur, Jalandhar, Punjab,INDIA, \\ *** (Department of Electronics Communication and Engineering, CTIEMT, Shahpur, Jalandhar, Punjab, INDIA,
}

\begin{abstract}
Vehicular Ad-hoc Network (VANET) is an extraordinary manifestation of MANET which is a vehicle to vehicle \& vehicle roadside remote correspondence system, where nodes in VANET include themselves as servers or clients for trading \& offering data. VANET (Vehicular Ad-hoc Network) could be another innovation that has taken gigantic consideration in the late years. Because of the quick topology the element cohort degreed incessant separation and it troublesome to style an efficient directing convention for steering data around vehicles, Vehicular Ad-hoc Network (VANET) could be a system of self sorted out vehicles and way side framework human movement with each one in turn over remote, with a read to support activity well being and intensity. Data exchanged over Vanets ordinarily assumes a significant part in movement well being. Such data ought to be right and truthful, as it rely on the requisition and verification is important administrations for activity security in Vanets. This paper is for the most part proposed to enhance the Quality of services (QOS) in vehicular Ad-hoc Network by breaking down parameters like throughput, End-to-End Delay, Average jitter, and so forth in conventions like ZRP, STAR and DSR by utilizing the Qualnet 5.0.1 test system.
\end{abstract}

Keywords : DSR, Qualnet, STAR, VANET, ZRP.

\section{INTRODUCTION}

VANET is essentially intended to give security related data, activity administration, and infotainment administrations. Security and movement administration oblige constant data and this passed on data can influence critical choices. Straightforward and viable security instrument is the significant issue of conveying VANET in broad daylight. Without security, a Vehicular Ad Hoc Network (VANET) framework is totally open to various assaults, for example, engendering of false cautioning messages and also concealment of genuine cautioning messages, consequently bringing on mischance's. This makes security a component of real concern in building such arranges. VANET are of prime imperativeness, as they are prone to be around the first business provision of specially appointed system innovation. Vehicles are the larger part of every last one of hubs, which are equipped for shaping self sorting out systems with no former learning of one another, whose security level is low and they are the most defenseless some piece of the system which could be ambushed effectively. The limit of VANET [1]innovation is high with an extensive variety of requisitions being conveyed in support of customers, business foundations, for example, toll courts, amusement organizations and additionally law requirement powers. On the other hand, without securing these systems, harm to life and property could be carried out at a more excellent degree, so security frameworks adroitly send and accept street data, for example, occurrences, ongoing movement data, or surface condition. In this respect, the viable directing system ought to be outlined with including such elements specified previously. Such a steering system ought to surety a stable and solid component over Vanets. Existing steering conventions, which are customarily intended for MANET, don't make utilization of the qualities of Vanets. Regarding nature's turf, the creator ought to consider that cars in diverse paths move at distinctive velocity, go down in diverse bearing. In such an element system, singular correspondence connections may not be a long lasting and the steering ways that shift habitually are insecure. In this paper, the execution assessment is contrasted and broke down and a few conventions like ZRP, STAR and DSR for VANET and the parameters like throughput, Average End-to-End Delay, Average jitter is gotten. The graphical client interface unit of VANET is sent to make the successful situation in the reenactment programming, in this manner all the investigation is effortlessly and flawlessly done. 


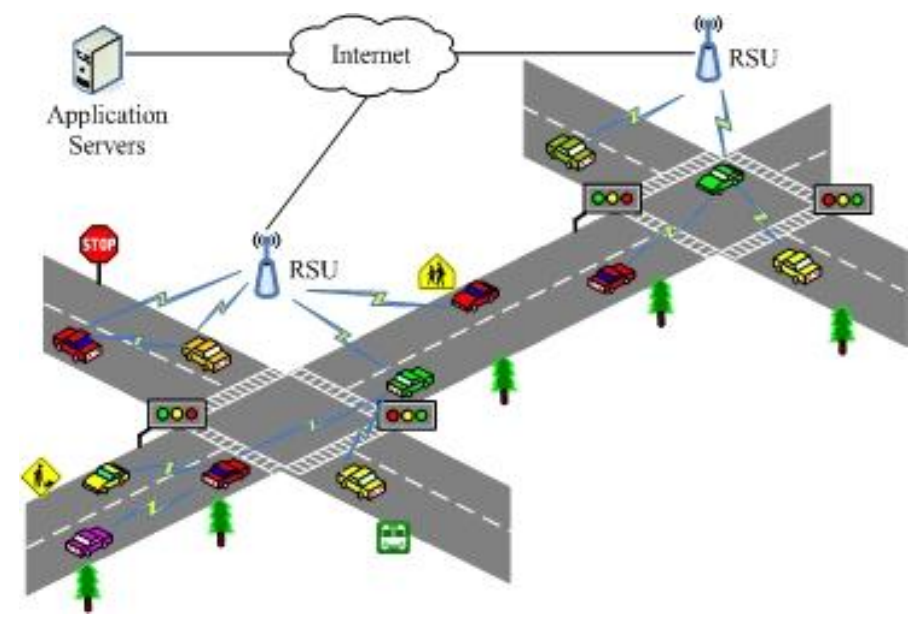

Fig 1:- Vehicular ad-hoc networks and some possible applications [5]

\section{Protocols Analysed In This PaPer}

\section{ZRP \\ 2. DSR \\ 3. STAR}

\subsection{Zone Routing Protocol}

The zone routing protocol will be provided by the frame work for other protocol. Neighbour discovery protocol will be used for the detection process. The size of the zone will be depending on strength of the signal, power which is available, node reliability etc. The Zone Routing Protocol[2] combines the benefits of the proactive and reactive approaches by maintaining an up-to-date topological map of a zone targeted on every node. Inside the zone, routes area unit directly obtainable. For destinations outside the zone, ZRP employs a route discovery procedure, which might like the native routing data of the zones. In ZRP, a proactive routing protocol (IARP) is employed in intra-zone communication and an inter-zone reactive routing protocol (IERP) is employed in inter-zone communication. Supply sends knowledge on to the destination if each area unit in same routing zone otherwise IERP reactively initiates a route discovery. Although the nature of the ZRP protocol seems to be hybrid, to show that it is a hierarchical protocol, it is emphasized to point out that the ZRP is in reality a flat protocol. In hierarchical network architecture, two different protocols are maintained for communication among every individual cluster's nodes and different clusters. The main difference here is that in the ZRP there is a one-to-one correspondence between nodes and routing zones, causing overlapping zones maintained by each individual node.

\subsection{DYNAMIC SOURCE ROUTING (DSR)}

It is a straightforward and exceptionally viable steering convention created just for the utilization of multi jump systems which will be convenient for portable hubs. It performs just on interest support. The DSR[3] convention will require each parcel to convey the entire location from source to objective. For huge systems the convention won't be extremely proficient. By expansion the measure of overhead help through a parcel it will naturally build the distance across system. It is created just for increment in the versatility rate at a reach of about 200 portable hubs that might be permitted. The point when change happens in a system it responds rapidly yet this convention has low overheads. This convention is made by two components yet it is worked just on interest premise. For lattice organizes there are three systems, in particular, Suppress course finding, Enable course revelation, Force course disclosure .Networks utilizing the DSR convention have been joined with the Internet. DSR can interoperate with Mobile IP, and hubs utilizing Mobile IP and DSR have consistently moved between WLANS, cell information administrations, and DSR portable specially appointed systems. The DSR convention is utilized essentially with the end goal of two fundamental instruments, Route Discovery and another is Route Maintenance, both of them join together for permitting the hubs to administer and find courses to goals in specially appointed systems. All the convention works completely on-interest, permitting the directing bundle overhead of DSR to consequently scale the vital and obligatory progressions required to respond for the progressions happening in the courses presently. Utilizing DSR, the system is totally having a place with self-organizing and designing, which fundamentally obliges no presence of system base or organization. The system hubs ought to chip in to send the bundles between one other to permit correspondence between various jumps between the hubs and not specifically to the remote transmission range which is available between each one in turn. The hubs in the system will join or leave the system, for remote 
transmission conditions like impedance change for the source. All directing has been consequently decided and it will be supported by a DSR steering convention. Since, the number or grouping of moderate jumps need to achieve any goal that might be changed at any moment of time the ensuing system topology may be extremely rich, dynamic and very arbitrary. The DSR convention permits hubs to powerfully find a source course crosswise over numerous system bounces to any objective in the specially appointed system. Each information parcel sends and afterward conveys its finished header, request the rundown of hubs through which the bundle must pass, permits the parcel directing to an inconsequentially circle free and maintaining a strategic distance from the need for state-of-the-art steering data in the halfway hubs through which the bundle is sent.

\subsection{SOURCE TREE ADAPTIVE ROUTING (STAR)}

The STAR protocol is proposed for utilization by nodes (static and versatile) in an ad hoc network or a web. A router in STAR[4] imparts to its neighbors the parameters of its source steering tree, which comprises of each one connection that the routers needs to achieve each known end (and location range) in the ad hoc network or web. To moderate transmission data transfer capacity and vitality, a routers imparts progressions to its source steering tree just when the router catches new goals, the likelihood of circling, or the likelihood of node disappointments or system parts. The STAR routing protocol could be run either by utilizing an ideal directing methodology (ORA) or by utilizing a minimum overhead routing methodology (LORA). The principal methodology tries to satisfy a prerequisite to give ideal ways between nodes as per a characterized metric and won't think about the volume of routing messages - truth be told its execution qualities will rely on upon the frequence of directing tables overhauls. Then again, the LORA approach as per its name strives to keep the overhead of directing messages to a base while relinquishing the optimality prerequisite of ORA. As LORA inclines towards an all the more steer correlation with on-interest routing conventions and likewise is additionally fascinating from an algorithmic perspective this overview concentrates on STAR-LORA. An intensive a talk of the ORA mode could be found.

\section{Performance Evaluation}

The protocol execution is investigated utilizing the Qualnet test system form 5.0.1. The simulation parameters utilized for simulating the situation of vehicular ad hoc network is demonstrated in the table 1.

Table.1: Simulation Parameters

\begin{tabular}{|c|c|}
\hline Parameter & Value \\
\hline Coordinate & 2200m X 1500m \\
\hline Application & CBR \\
\hline VERSION & QUALNET 5.0.1 \\
\hline ROUTING PROTOCOL & ZRP, STAR AND DSR \\
\hline Network & IPv4 \\
\hline MAC & IEEE $802.11 \mathrm{e}$ \\
\hline NO OF NODES & 20 to 100 \\
\hline
\end{tabular}

\section{SIMULATION SCENARIO}

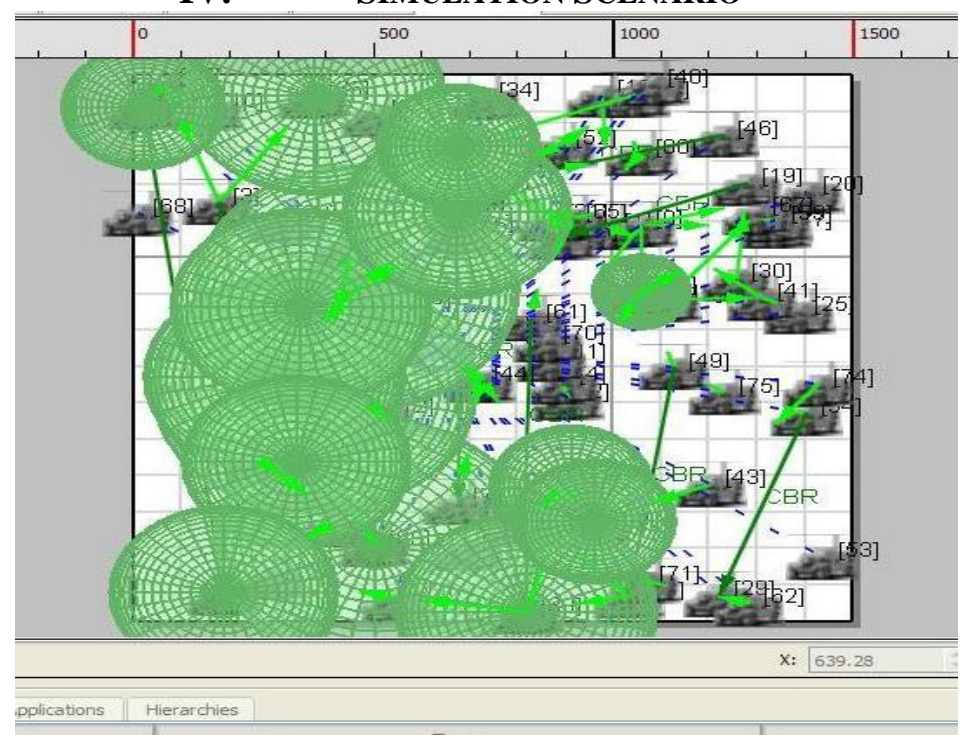

Fig 2: A Scenario illustrating the analysis of ZRP, STAR AND DSR protocols in Vehicular Adhoc Network 


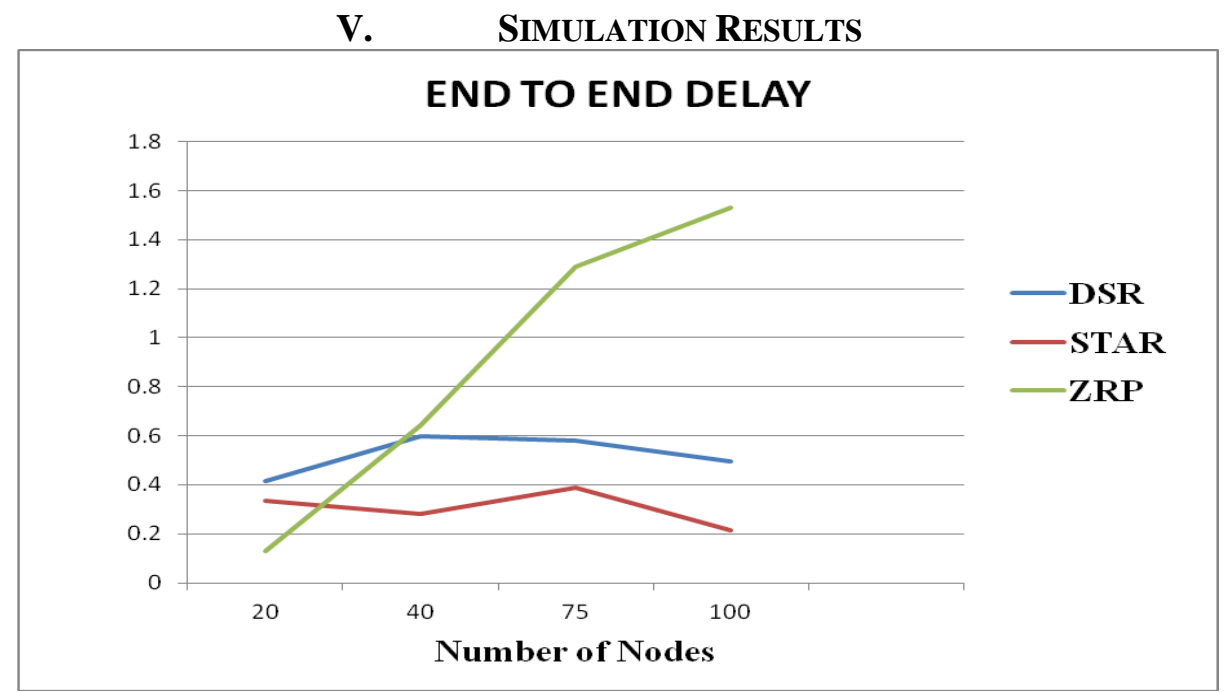

Fig 3. Analysis of End To End Delay

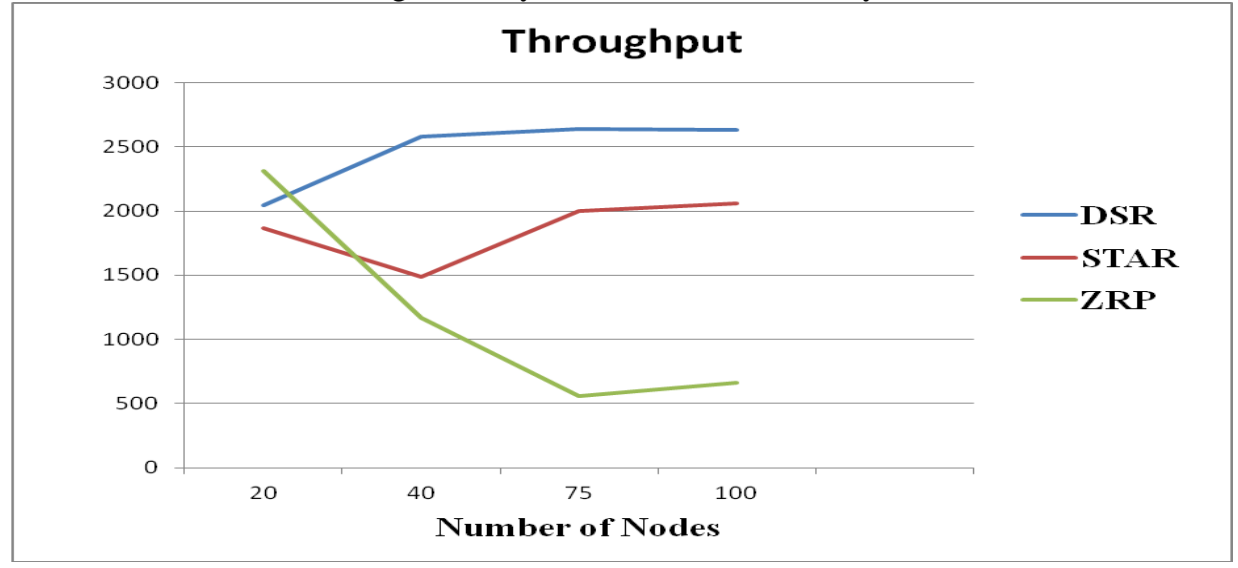

Fig 4. Analysis of Throughput

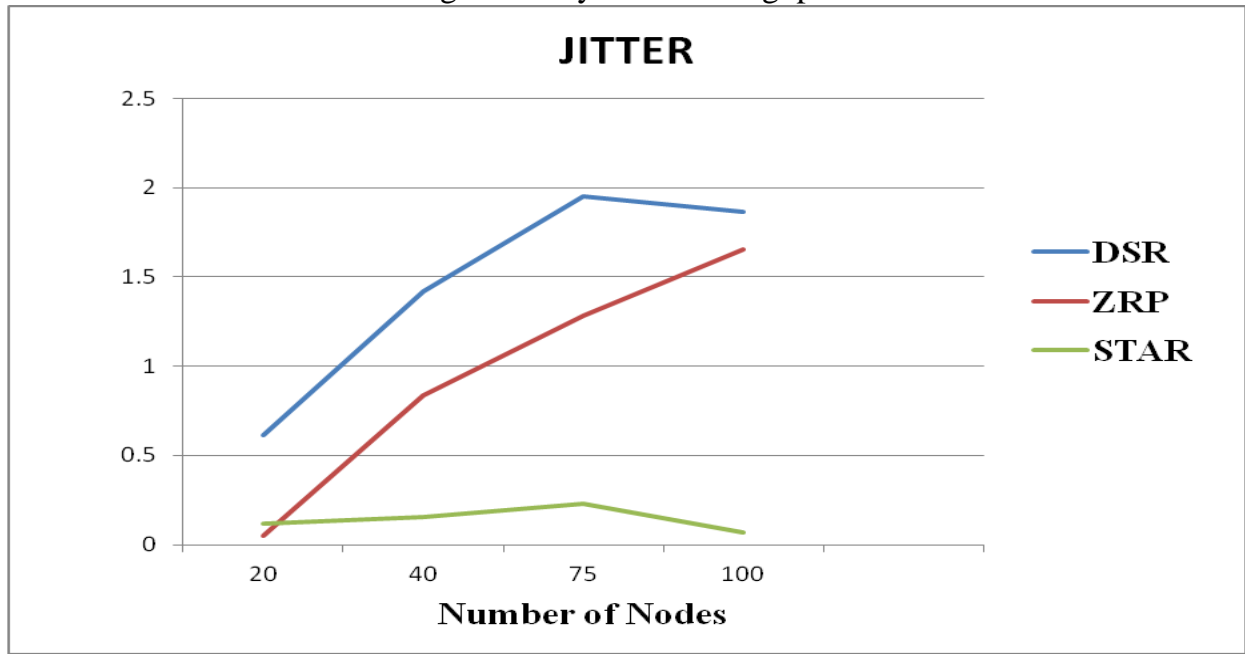

Fig 5. Analysis of Jitter

VI. RESULTS AND DISCUSSIONS

This section describes the details of the performance and results evaluation of the simulations done for the ZRP, STAR and DSR routing protocol.

\subsection{AVERAGE END TO END DELAY}

Fig 3. shows average end to end delay with number of nodes (vehicles) varying from 20 to 100 for DSR, STAR and ZRP protocol. The basic difference between STAR and DSR is very less throughout the nodes variation. Average end to end delay of ZRP is higher than both STAR and DSR. But after number of nodes 
increased by 30, STAR is giving lesser end to end delay than DSR. In case of ZRP, initially it is giving lesser end to end delay as compared to DSR and STAR. After number of nodes increases 30, there is consistently increment in the value of end to end delay. In ZRP protocol routes discovery is slow. Inter-Zone routing (IERP) is responsible for this work. If link is broken during route instead of using an alternative path, it uses the local route repair to form a new route as in some reactive protocol. In some cases this route can be pretty long (in number of hops) and continues to send the data packets along the long route. Therefore, the end-to-end delay increases for these data packets, resulting in increased average end-to-end delay for all data packets.

\subsection{Throughput}

Fig 4. shows throughput with number of nodes varying from 20 to 100 nodes for STAR, DSR and ZRP routing protocol. Throughput of DSR is better than STAR and ZRP.As the number of nodes is increasing, the value of throughput is also increasing which shows that DSR supports scalability. STAR also shows higher throughput as compared to ZRP. Up to 35 nodes there is slightly difference in the throughput of both STAR and ZRP. But after this, STAR is showing consistently increment in throughput value. Whereas DSR throughput is remains constant after number of nodes become 80 . So DSR only routing protocol which supports scalability as the numbers of nodes are increasing its throughput also increased.

\subsection{JITTER}

Fig 5. shows Jitter with number of nodes varying from 20 to 100 nodes for STAR, DSR and ZRP routing protocol. Jitter of STAR is lesser than both DSR and ZRP .As the number of nodes is increasing, the value of jitter is also increasing for both ZRP and DSR.

\section{CONCLUSION}

Initially, various routing protocols are surveyed in this paper. Keeping challenges and issues of VANETs in our mind, we have selected three routing protocols to be compared based on their simulation performance. In this paper, the performance of Position Based routing protocol (STAR), Reactive routing protocol (DSR) and Hybrid routing protocol (ZRP) is evaluated using QUALNET 5.1 simulator on urban scenario in which nodes are moving in one direction. The performance of the protocols was measured with respect to metrics like jitter, end to end delay and throughput on the basis of varying number of nodes. Simulations were carried out with identical topologies and running different protocols on the moving vehicles. The results of the simulation indicate that performance of the STAR protocol is superior to both DSR, ZRP protocols. It is also observed that the performance is better especially when the number of nodes is increased. In case of throughput DSR perform better than both ZRP and STAR. But in other two performance metrics STAR outperformed in Jitter and End to End Delay

\section{REFERENCES}

[1] Bijan Paul, Md. Ibrahim, Md. Abu Naser Bikas " VANET Routing Protocols: Pros and Cons" International Journal of Computer Applications (0975 - 8887) Volume 20- No.3, April 2011

[2] Z. J. Haas, "The Zone Routing Protocol", Sixth WINLAB Workshop on Third Generation Wireless information Networks, New Brunswick, NJ, Nov. 1997.

[3] D. Johnson, B.D.A. Maltz, Y.C.Hu, "DSR", by International Journal of Advance in Science and Technology, 2004, pp.64-69

[4] Seet, B.-C., "A-STAR: A Mobile Ad Hoc Routing Strategy for Metropolis Vehicular Communications." 1980.

[5] www.google.co.in 